\title{
Sequence analysis of earthworm hemolysins
}

\author{
Simone König ${ }^{\mathrm{a}, *}$, Frank Wagner $^{\mathrm{b}}$, Ellen Kauschke ${ }^{\mathrm{c}}$ and Ines Eue ${ }^{\mathrm{d}}$ \\ a Integrated Functional Genomics, IZKF University of Münster, Germany \\ ${ }^{\mathrm{b}}$ Zoological Institute, University of Greifswald, Germany \\ ${ }^{c}$ Institute of Anatomy, University of Greifswald, Germany \\ ${ }^{\mathrm{d}}$ In den Wolfseichen 5, Lohmar, Germany
}

\begin{abstract}
Annelids are capable of defending themselves from pathogens and of recognizing degenerated self tissue. These reactions require specialized immune mechanisms that are effected by proteins and cellular reactions. Hemolytic proteins are the most striking humoral defense molecules in the earthworm Eisenia fetida. Beside their hemolytic activity these proteins possess agglutinating, antibacterial, cytotoxic, and clotting properties. Hemolytic proteins both from coelomocytes $\left(\mathrm{CL}_{39,41}\right)$ and coelomic fluid $\left(\mathrm{H}_{1-3}\right)$ of wildtype E. fetida were isolated and assigned to fetidin and lysenin using mass spectrometry and bioinformatic tools. Glycosylation was found for $\mathrm{H}_{1}$. In silico analyses of the hemolysins revealed two hemolysin isoforms and consensus sites for $\mathrm{N}$-glycosylation and peroxidases proximal heme-binding ligand.
\end{abstract}

Keywords: Eisenia fetida, hemolysins, mass spectrometry, isoforms, glycosylation, hydrogen peroxide

\section{Abbreviations}

$\mathrm{CF}$, coelomic fluid; $\mathrm{CL}$, coelomocyte lysate; $\mathrm{CL}_{39,41}, 39$ and $41 \mathrm{kDa}$ hemolysins isolated from coelomocyte lysate; $\mathrm{H}_{1-3}$, three hemolytic CF proteins; LRP, lysenin-related protein; MS, mass spectrometry.

\section{Introduction}

Earthworms, and, in fact all annelids, are interesting immunological study objects. They are capable of defending themselves from pathogens and of recognizing degenerated self tissue. These reactions require specialized immunological mechanisms. Hemolytic proteins are the most striking humoral defense molecules in the earthworm Eisenia fetida. They, as well as several bacterial toxins, toxins secreted from invertebrate cells, and even perforin and components of the complement cascade in vertebrates, belong to a family of phylogenetically very old, barrel-like pore forming, membrane attacking proteins. Hemolytical and agglutinating proteins in E. fetida were originally described by DuPasquier and Duprat [3]. Beside their hemolytic activity these proteins possess agglutinating [4,6,14], antibacterial [11,13], cytotoxic [7] and clotting [15] properties. We isolated and analysed hemolytic proteins both from $\mathrm{CL}\left(\mathrm{CL}_{39}\right.$ and $\left.\mathrm{CL}_{41}\right)$ and $\mathrm{CF}\left(\mathrm{H}_{1-3}\right)$ of wildtype E. fetida as illustrated in Fig. 1, and assigned them to fetidin and lysenin [9], which have been described earlier [10,12]. The results supported the concept that the hemolytic proteins in E. fetida originate from chloragocytes and are secreted into CF [9]. Here, we present sequence analyses based on the MS data using similarity, pattern, and profile searches.

\footnotetext{
${ }^{*}$ Corresponding author: Dr. Simone König, Integrated Functional Genomics, Von-Esmarch-Str. 56, 48149 Muenster, Germany. Tel.: +49 (0)251 835 7164; Fax: +49 (0)251 835-5651; E-mail: koenigs@ uni-muenster.de.
} 

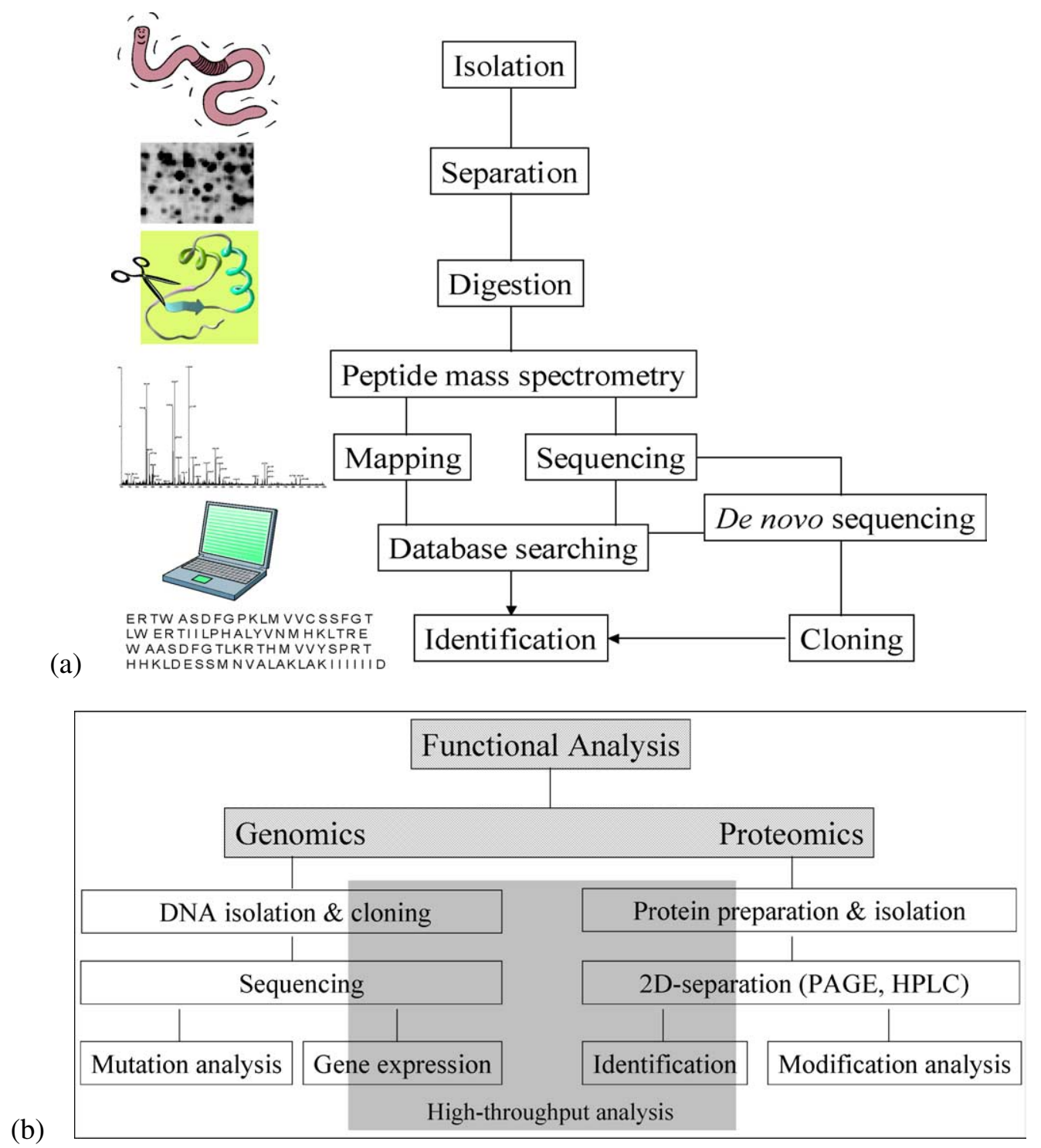

Fig. 1. Earthworm proteins were subjected to MS-based analysis which is part of the proteomics toolkit [9]. (a) Thereby, proteins are separated using gel electrophoresis and digested tryptically in the gel spot of interest. Peptides are then eluted and subjected to mass mapping or sequencing. MS data allow the identification of known proteins from databases. In case of unknown proteins MS contributes detailed structure information. (b) Functional analyses require knowledge on the genomic and proteomic level. In both areas high-throughput techniques are available, which generate huge data amounts for gene expression or protein identification experiments. However, in order to access modifications, mutations, and isoforms, which are of uttermost importance for functional studies, the methods need to be refined and optimised for the respective analysis task.

\section{Experimental}

Proteins were isolated as described [9] and illustrated in Fig. 2. Database, similarity (BLAST2 [1]), pattern and profile (Prosite) searches have been performed using public access of the EXPASY server searching SwissProt (University of Geneva and European Bioinformatics Institut) and the NCBI Entrez server searching Genbank (National Center of Bioinformatics, Bethesda, MD, USA). 


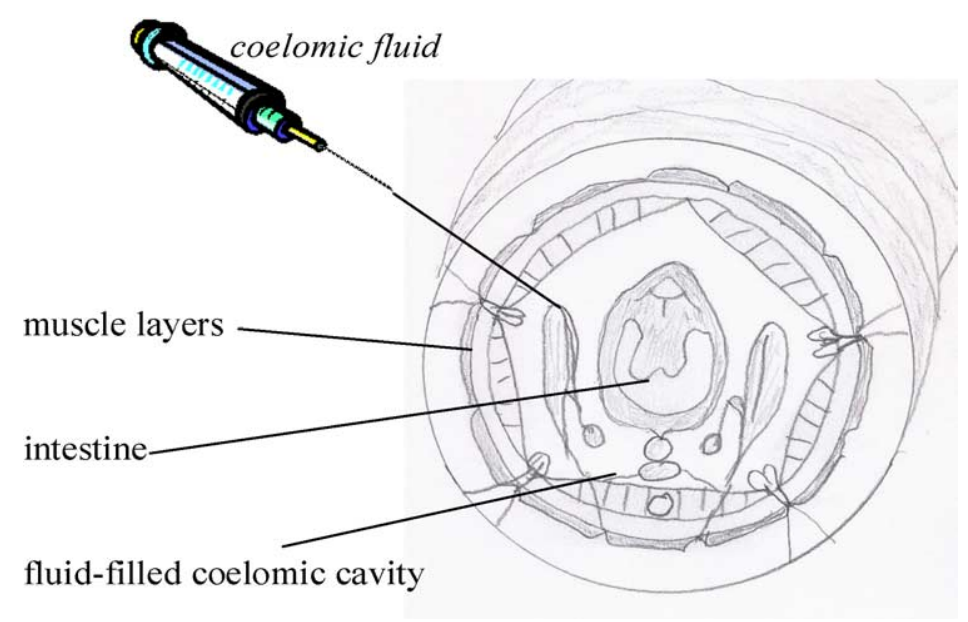

Fig. 2. The source of hemolysins was CF of adult E. fetida harvested by puncturing the coelomic cavity with a very thin glass capillary.

\section{Results and discussion}

Eisenia is currently not subject of extensive sequencing projects, although efforts have been started to sequence Lumbricus rebellus (http://convoluta.cap.ed.ac.uk/Lumbribase/lumbribase/lumbribase.html). Therefore, only 5 proteins can be found in SwissProt and 74 in Genbank among hundreds of thousands of entries. Only the fact that relevant earthworm proteins have been analysed at the genomic level [10, 12] allowed us to interpret the sequencing data of the CL and CF hemolysin proteins [9]. We had noted that the proteins share large parts of their sequences as is demonstrated in Fig. 3. There are four entries in Genbank, which are extremely similar, and those are fetidin (U02710, $\mathrm{CL}_{39}$ [10]), lysenin (D85846, $\mathrm{CL}_{41}$ [12]), and lysenin-related proteins 1 and 2 (D85848 and D85847 [12]). Fetidin and lysenin are to $90 \%$ and fetidin and LPR2 to $75 \%$ identical, so that the analytical challenge was to find the few peptides which are different. Protein sequence analysis also showed that the amino acid sequences of LPR 1 and fetidin are completely identical (Fig. 3b). However, detailed analysis at the genomic level (Figs 3a, 4) revealed that those proteins are isoforms. Guanine 621 in fetidin is replaced by adenine 672 in LPR 1 (the position being defined by the length of the submitted mRNA sequences). Nevertheless, this mutation does not have an effect on the translation, since both AAA and AAG code for Lys178 (Fig. 3b).

For N-glycosylation of the high-mannose type, which was indicated to be present by the MS results ([9], Fig. 4), the signature can be found at Asn250 (Fig. 3b). This consensus sequence is also found in lysenin. LPR2 does not have this site, but there is a N-glycosylation pattern towards the $\mathrm{N}$-terminal end at Asn33. However, the particular pattern $\mathrm{N}-\mathrm{x}-\mathrm{S} / \mathrm{T}$ is found often, due to its length, without functional relevance. More interesting in that respect is the finding of a peroxidases proximal heme-ligand signature in all three hemolysins (Fig. 3b). The pattern is [DET]-[LIVMTA]-xx-[LIVM]-[LIVMSTAG][S AG]-[LIVMST AG]- $\boldsymbol{H}$-[STA]-[LIVMFY] with variable amino acids residues in brackets and found residues italicized. In this pattern His is the proximal heme-binding ligand according to Prosite rules [2, $5,8]$. Heme-binding peroxidases carry out a variety of biosynthetic and degradative functions using hydrogen peroxide as the electron acceptor. Hydrogen peroxide is a toxic substance which causes dramatic intracellular degradation effects, if it accumulates in the cell and is not metabolized. In so far a detoxification function of earthworm hemolysins can be speculated and needs to be investigated. 
similarity

protein-based

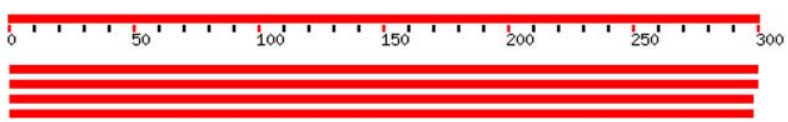

$\begin{aligned} & m R N A \text {-based } \\ & \text { lysenin D85846 }\end{aligned}$
LPR1 D85848
fetidin U02710

(a)

LPR2 D85847

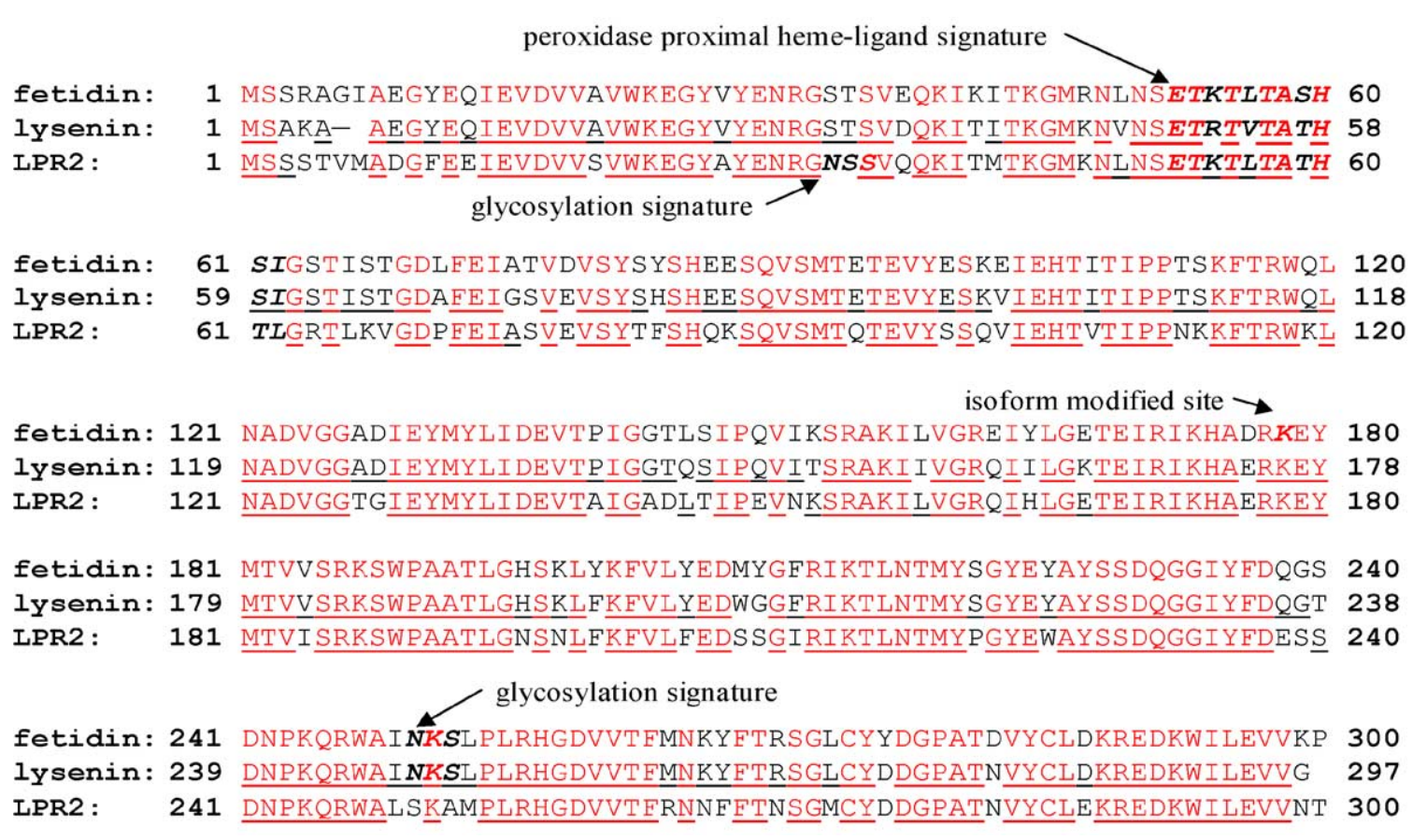

Fig. 3. (a) BLAST similarity analysis of Eisenia hemolysins. Top result: protein-based; bottom result: nucleotide-based. Bold lines indicate high similarity. (b) Comparison of protein sequences (fetidin, lysenin, and LPR2). Consensus sites are marked. (c) Comparison of mRNA sequences of 1) lysenin, 2) LPR1, 3) fetidin, 4) LPR2. Coding regions are indicated with arrows as well as the mutation A672 in LPR1. (Note: for Fig. 3(c) see next two pages.)

\section{Conclusion}

Hemolytic proteins both from $\mathrm{CL}\left(\mathrm{CL}_{39}\right.$ and $\left.\mathrm{CL}_{41}\right)$ and $\mathrm{CF}\left(\mathrm{H}_{1-3}\right)$ of wildtype $E$. fetida were carefully isolated by puncturing the coelomic cavity minimizing protein cross-contamination between cells and fluid. MS analysis assigned the hemolysins to fetidin and lysenin and high-mannose $\mathrm{N}$-glycosylation was detected for CF fetidin. In silico sequence analyses of four Eisenia hemolysins revealed high similarity among them, in fact, the two sequences of LPR1 and fetidin only differ in one base with no effect on translation. Consensus sites for N-glycosylation are pointed out in this study, but it is yet unknown whether glycosylation does occur at those sites. This is also true for the peroxidases proximal hemebinding ligand site discovered towards the $\mathrm{N}$-terminal site of the protein, although this pattern is highly 
1) 1

2)

4)

1) 61

2) 61

3) 18

1) 120

2) 120

3) 68

4) 139

4) 100

1) 171

2) 178

3) 127

4) 157

1) 231

3) 187

4) 217

1) 291

2) 298

3) 247

4) 277

1) 351

2) 358

3) 307

4) 337

1) 411

2) 418

3) 367

4) 397

1) 471

4) 457

1) 531

2) 538

3) 487

4) 517

1) 591

2) 598

3) 547

1) 651

2) 658

3) 607

4) 637

1) 711

718

3) 667

4) 697

1) 771

1) 831

2) 838

3) 787

4) 817

1) 891

2) 898

3) 847

3) 847

1) 951

2) 958

(c) 3) 907

ctcagatgcagttattgtatctaataggcatctcggtcttccgctggtttctcgcagata 60 .......................................... 60 ..........t..tct........... 40

catcaaagactagaagaggccatcaagatcctgtt-gaattgagtgcactcctgtttgc 119

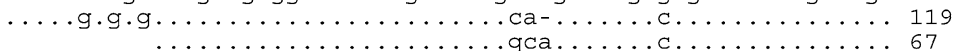

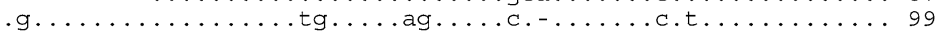

--t-tcagaaacttgtgagcgatgtcggctaaagcag------cagagggatatgaacag 170 - -ca.....t..................gaatcg........... 177

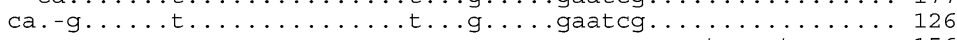

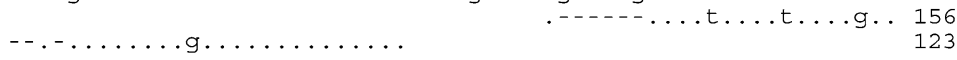

atagaagtagatgtggtggcagtatggaaagaaggctatgtatacgaaatcgcggaagc 230

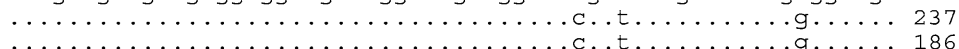

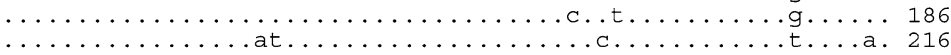

accagtgtggatcagaagatcacaataacaaaaggcatgaaaaatgtgaattcagaaaca 290

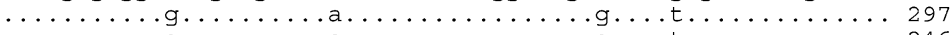

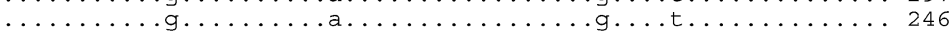

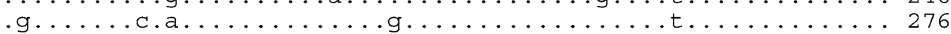

aggacagtgactgctacgcatagtattggttctactattagcactggagatgcattcgaa 350

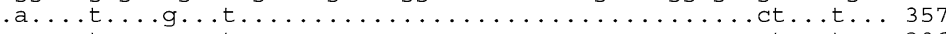

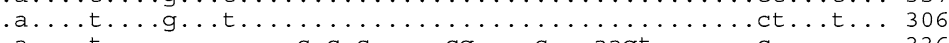

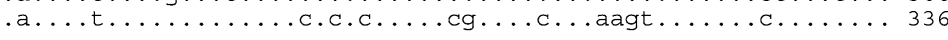

attggaagcgtggaggttagctacagccattcacatgaagaatcccaagttagcatgacg 410


.a.c...........t...c.tt..................... 396

gaaactgaagtttatgaatcaaaggtgatcgaacacactataacgattccacctacttca 470

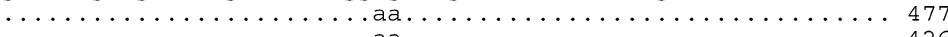

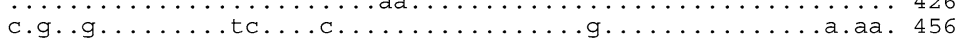

aattcacaagatggcaactgaatgctgacgttggtggagcggatattgaatacatgtat 530

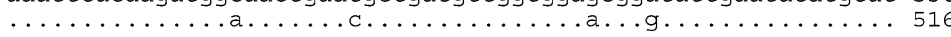
ttgattgatgaagtcacacccataggagggactcagagtattccacaggtcatcacaagt 590

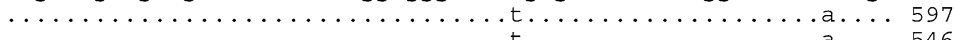

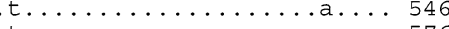
cgggctaaaattatagttggccgacagataatccttggaaaaacagaattcgaattaag 650

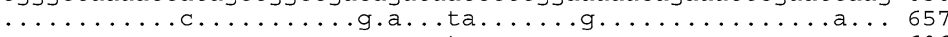

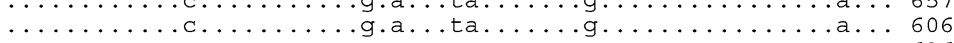
$\ldots \ldots \ldots \ldots$. . . . . . . . . . . . . . . . 636

catgcagaaaggaaggagtacatgacagtcgtttcaagaaaaagttggccagctgcaact 710

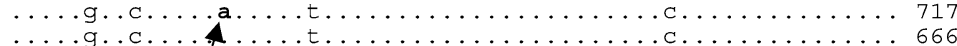

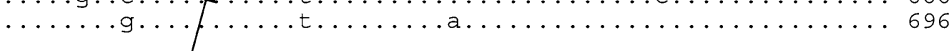
cttggacatagcaaactttcaagtttgtgctctatgaagattgggggggatttcgaatt 770



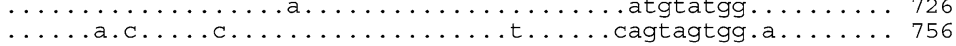

aaacgctgaacaccatgtattcgggctatgagtatgcctattcctctgatcaaggagga 830

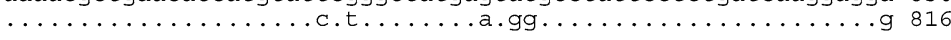
atctactttgatcagggtactgataatccgaaacagcgctgggcaatcaataagtcattg 890

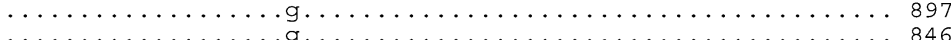

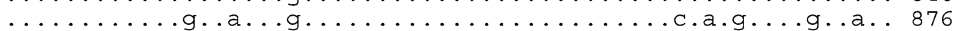

cctctcgtcatggtgacgtagtcacctcatgaataagtacttcactcgcagtgggctg 950

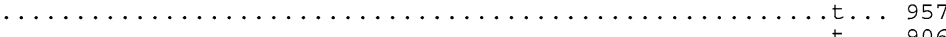
...

tgctacgatgatggaccggcaacaaacgtgtactgtctggacaaacgtgaagacaagtgg 1010

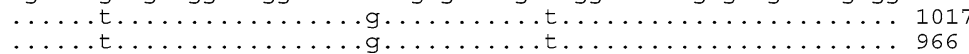

Fig. 3. (Continued). 


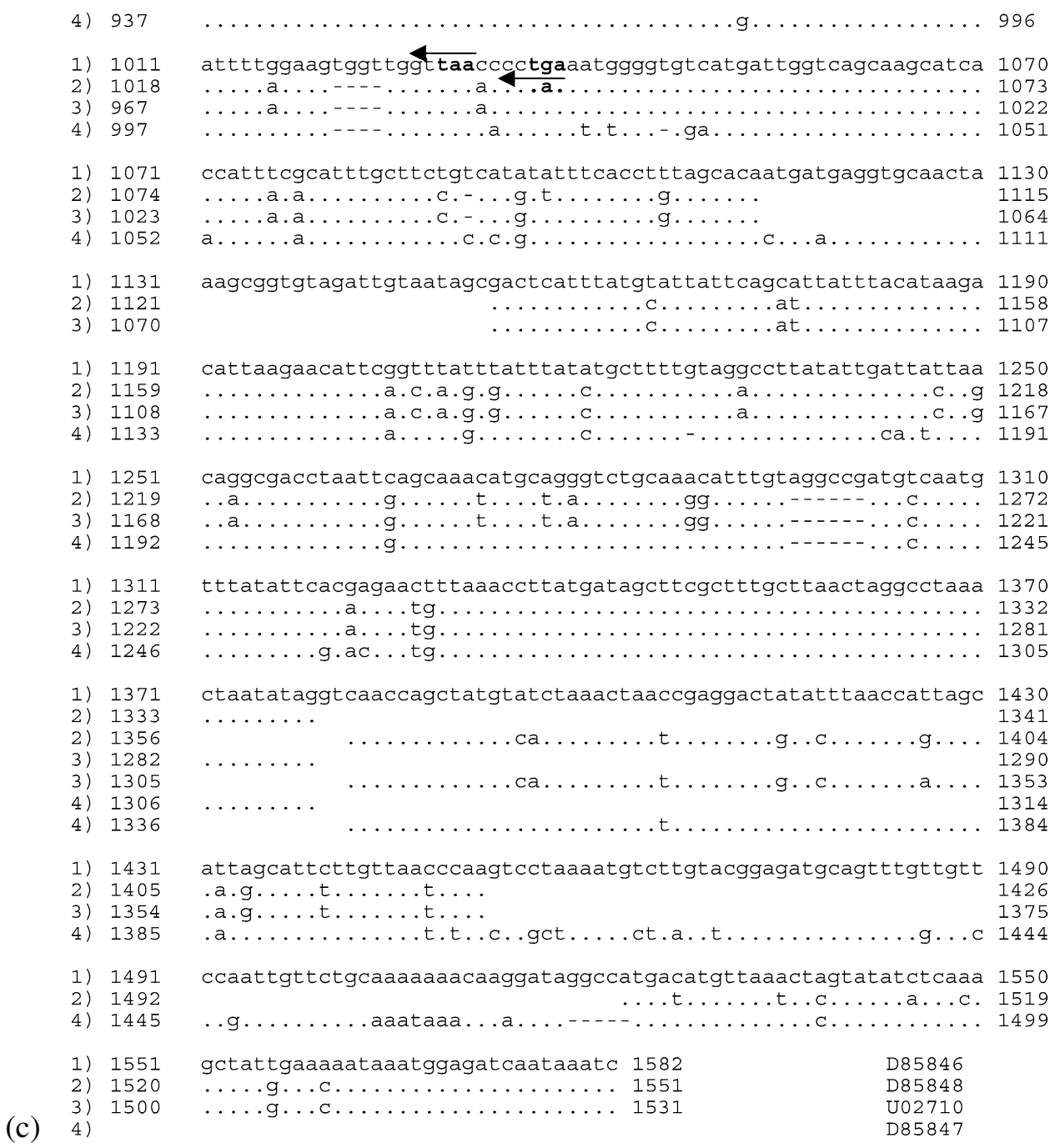

Fig. 3. (Continued).

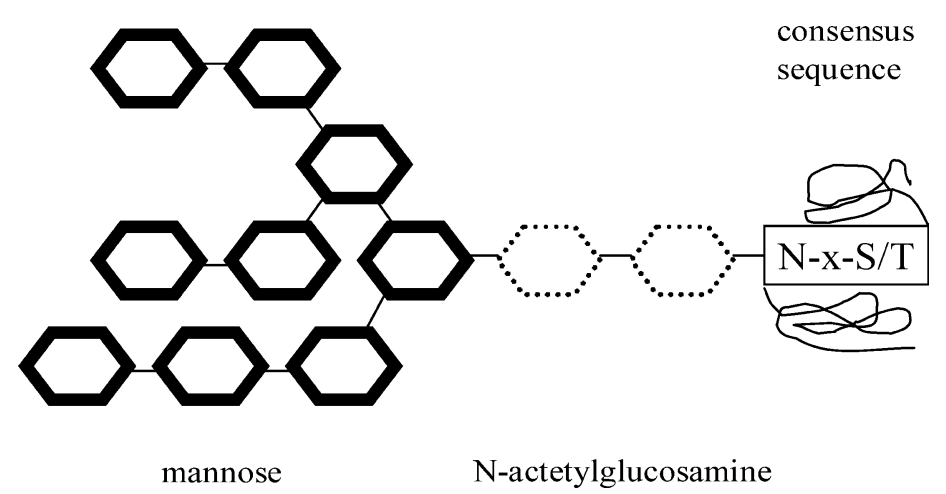

Fig. 4. Basic core structure for Asn-glycosylation of the high-mannose type. The number of mannose residues is variable. $\mathrm{X}$ is any amino acid except Pro. 
specific. The data initiate experiments to study those findings especially with respect to a hemolysin involvement in hydrogen peroxide detoxification.

\section{Acknowledgements}

S. König acknowledges the use of the resources of the Interdisciplinary Center for Clinical Research (Medical School, University of Münster, grant from Federal Ministry of Education and Research (Fö.01KS9604/0)); and F. Wagner a scholarship of county Mecklenburg-Vorpommern.

\section{References}

[1] S.F. Altschul, T.L. Madden, A.A. Schaffer, J. Zhang, Z. Zhang, W. Miller and D.J. Lipman, Gapped BLAST and PSIBLAST: a new generation of protein database search programs, Nucleic Acids Res. 25 (1997), 3389-3402.

[2] J.H. Dawson, Probing structure-function relations in heme-containing oxygenases and peroxidases, Science 240 (1988), 433-439.

[3] L. Du Pasquier and P. Duprat, Aspects humoraux et cellulaires d'une immunité naturelle non spécifique chez l'oligochète Eisenia fetida (SAV.), CR Acad. Sci. Paris 266 (1968), 538-542.

[4] I. Eue, E. Kauschke, W. Mohrig and E.L. Cooper, Isolation and characterization of earthworm hemolysins and agglutinins, Dev. Comp. Immunol. 22 (1998), 13-25.

[5] B. Henrissat, M. Saloheimo, S. Lavaitte and J.K.C. Knowles, Structural homology among the peroxidase enzyme family revealed by hydrophobic cluster analysis, Proteins 8 (1990), 251-257.

[6] E. Kauschke and W. Mohrig, Occurrence of bacterioagglutinating, hemagglutinating and hemolytical compounds in the coelomic fluid of different species of European Earthworms (Annelida, Lumbricidae), Zool. Jb. Physiol. 92 (1987), 467477.

[7] E. Kauschke and W. Mohrig, Cytotoxic activity in the coelomic fluid of the annelid Eisenia foetida (SAV.), J. Comp. Physiol. 175 (1987), 77-83.

[8] S. Kimura and M. Ikeda-Saito, Human myeloperoxidase and thyroid peroxidase, two enzymes with separate and distinct physiological functions, are evolutionarily related members of the same gene family, Proteins 3 (1988), 113-120.

[9] S. Koenig, F. Wagner, E. Kauschke, J. Peter-Katalinic, E.L. Cooper and I. Eue, Mass spectrometric analyses of CL39, CL41, and $\mathrm{H} 1, \mathrm{H} 2$, and $\mathrm{H} 3$ confirm identity with fetidin and lysenin produced by earthworm leukocytes, Dev. Comparative Immun. 27 (2003), 513-520.

[10] M. Lassegues, A. Milochau, F. Doignon, L. Du Pasquier and P. Valembois, Sequence and expression of an Eisenia-fetidaderived cDNA clone that encodes the 40-kDA fetidin antibacterial protein, Eur. J. Biochem. 246 (1997), 756-762.

[11] A. Milochau, M. Lassègues and P. Valembois, Purification, characterization and activities of two hemolytic and antibacterial proteins from coelomic fluid of the annelid Eisenia fetida andrei, Biochim. Biophys. Acta 1337 (1997), 123-132.

[12] Y. Sekizawa, T. Kubo, H. Kobayashi, T. Nakajima and S. Natori, Molecular cloning of cDNA for lysenin, a novel protein in the earthworm Eisenia foetida that causes contraction of rat vascular smooth muscle, Gene 191 (1997), 97-102.

[13] P. Valembois, P. Roch, M. Lassègues and P. Cassand, Antibacterial activity of the hemolytic system of Eisenia fetida, J. Invert. Pathol. 40 (1982), 21-27.

[14] P. Valembois, P. Roch and M. Lasségues, Simultaneous existence of hemolysins and hemagglutinins in the coelomic fluid and cocoon albumen of the earthworm Eisenia fetida andrei, Comp. Biochem. Physiol. 78(A) (1984), 141-145.

[15] P. Valembois, P. Roch and M. Lassègues, Evidence of plasma clotting system in earthworms, J. Invert. Pathol. 51 (1988), $221-228$. 


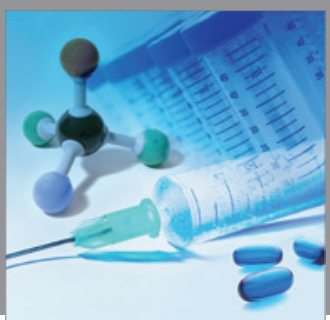

International Journal of

Medicinal Chemistry



Carbohydrate Chemistry

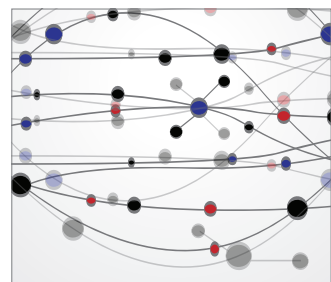

The Scientific World Journal
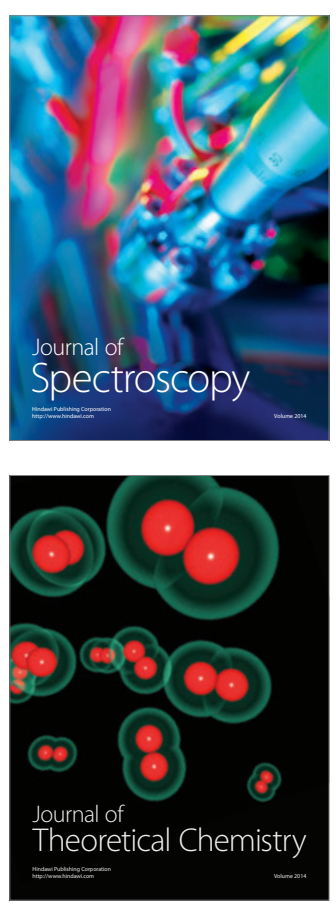
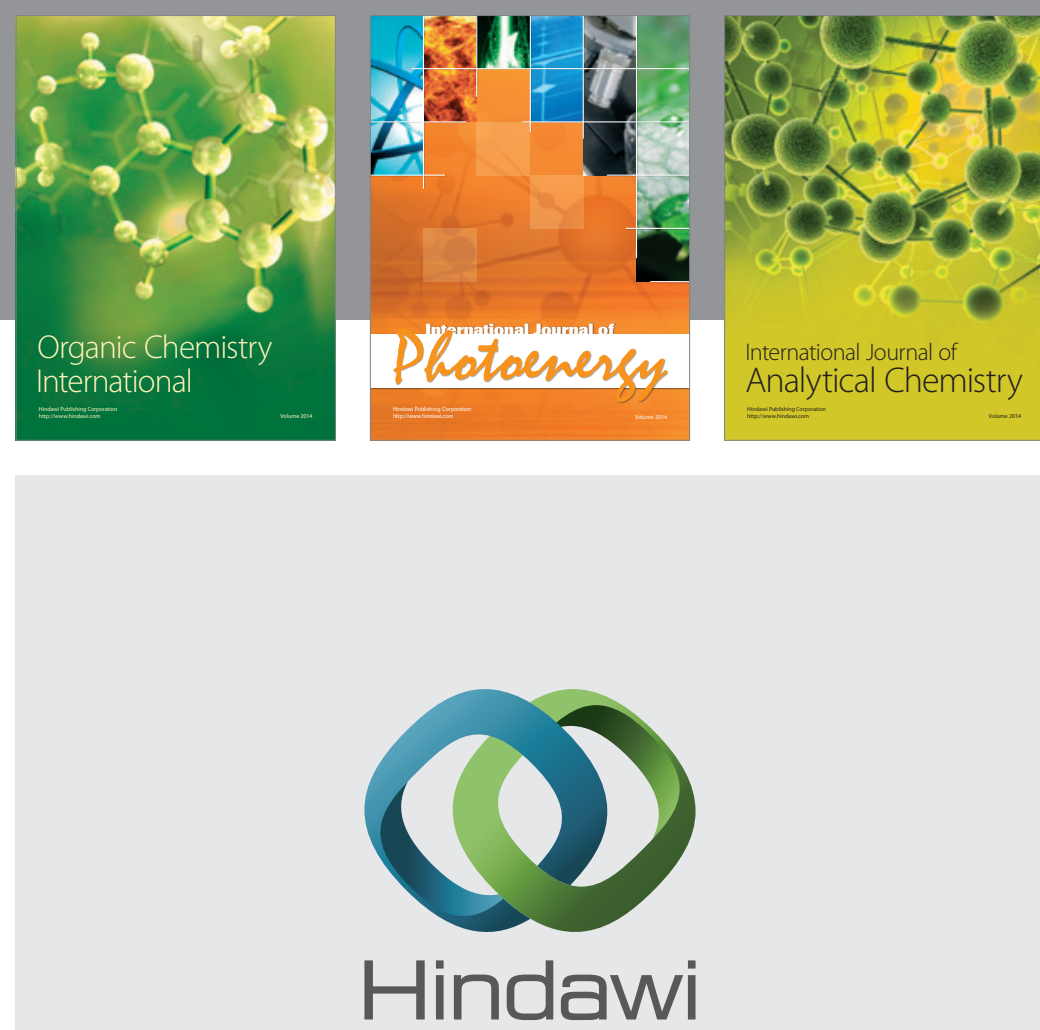

Submit your manuscripts at

http://www.hindawi.com
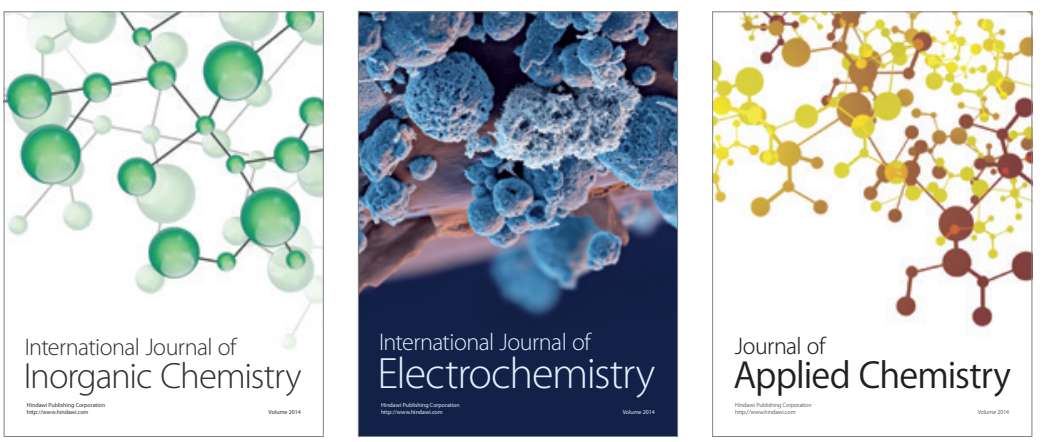

Journal of

Applied Chemistry
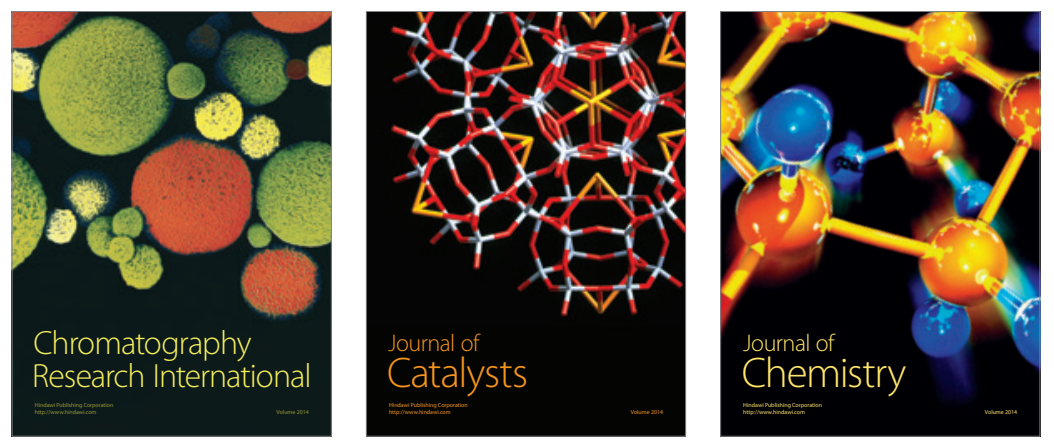
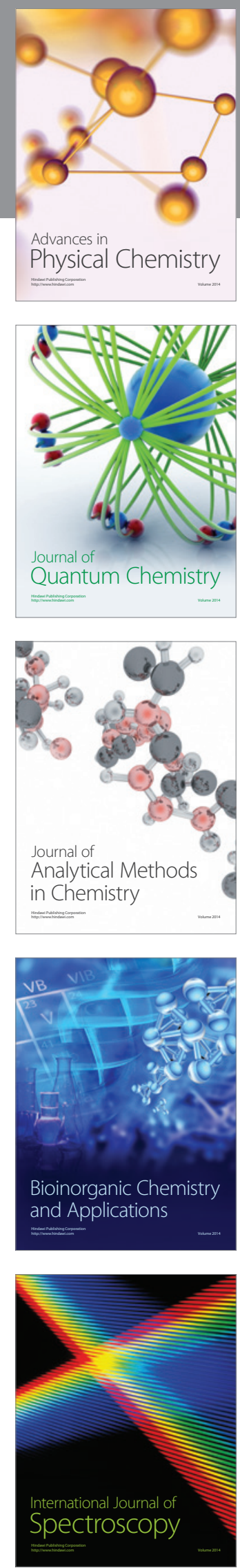